\title{
Adaptive User-Centered Design for Safety and Comfort of Physical Human Nursing - Care Robot Interaction
}

\author{
Minghui Sun ${ }^{1,2}$, Hiromichi Nakashima ${ }^{2}$, Shinya Hirano ${ }^{2}$, Kazuya Matsuo ${ }^{2}$, \\ Ming Ding ${ }^{2}$, Chang'an Jiang ${ }^{2}$, Toshiharu Mukai ${ }^{2}$, and Guihe Qin ${ }^{1}$ \\ ${ }^{1}$ College of Computer Science and Technology, Jilin University, \\ Changchun, 130012, China \\ ${ }^{2}$ RIKEN RTC, 2271-130, Nagoya, 463-0003, Japan \\ sunmh.cn@gmail.com
}

\begin{abstract}
Nowadays serving robots are more and more popular in human society. However, most of them are designed for the special people or for the special scenario. There is little robot designed to apply appropriate interface for different people that can accommodate age-related and body-related in physical interaction. We propose that user-centered design should be used in physical Human-robot interaction. In this research, we take a nursing-care robot as an example. Based on the results of the experiment, we proved that the distance between two arms of nursing-care robot, which affected the comfort and safety of patient, should be applied by different patients with different body length. We try to build the adaptive human robot interface based on the physical properties of people, such as body length. This study is an attempt to explore the adaptive human robot interaction and contributes to giving insights and implications for the future design of general serving robot.
\end{abstract}

Keywords: Physical human robot interaction, nursing-care robot, safe transferring.

\section{Introduction}

With the advent of an aging society, robots are popular in human society and expected to assist the human being. Many researchers explored the physical human robot interaction (PHRI) in many areas, for example, industry, medical, welfare, dancing, music, homework assistance, nursing support and so on. For example, Partner Ballroom Dance Robot (PBDR) [1] can predict the steps of the partner based on body movement and react on wheels. $\mathrm{CB}^{2}[2]$ is used for safe operation and behavior learning and try to understand the human developmental process of cognitive functions through mutual feedback. Robovie-IV [3] was designed to "socially" interact with people in daily lives. However, most of these robots are designed for the special people or for the special scenario. There is little research has been targeted at adaptive user-centered design for ordinary people in public environment. Sekmen and Challa [4] built an HRI system and predicted the behaviors and preferences of the people with a Bayesian learning method. In this research, adaptive user-centered interface is explored by natural properties of the human being. 
Human being has two properties in the nature. They are physical property and social property. Physical properties include body length, thigh length, shank length, arm length, body weight, disability, and waist strength and so on. Social properties include career and aging and so on. User-centered design should be used to implement the physical Human-robot interaction (PHRI). Many PHRI happens in hospital. For example, doctor can lead a robot to a room hand by hand and then robot can lift and move the patient from one place to the other. Robot also can guide the patients' friends to the place where the patient is. During PHRI, both social and physical communications occur. Robot must adapt the interaction, such as leading, following, or lifting, based on the social properties of people, such as patient, doctor and patient's friend. At the meantime, according to the physical properties (aging, body height, body weight) of patient, different communication strategies should be applied while lifting and moving a patient. For example, when lifting an infant (shown in Figure 1a), we must hold her/his neck carefully because infant's neck is very weak. For an adult, princess lifting motion is often used (shown in Figure 1b). Moreover, robot should adjust the distance between two arms based on the different patients.
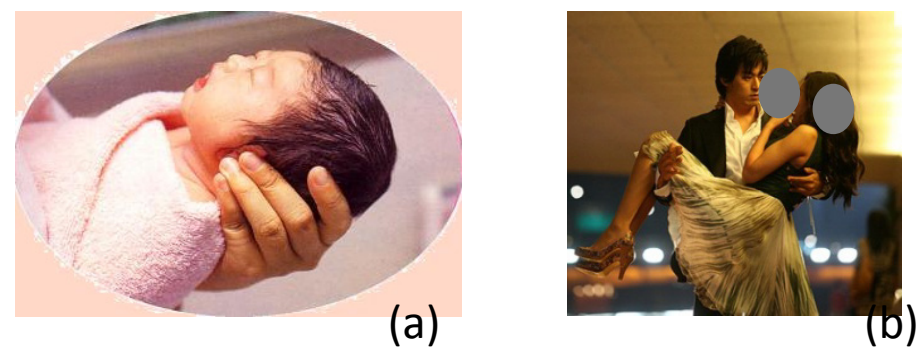

Fig. 1. (a) lifting an infant; (b) lifting an adult

In this research, we take a nursing-care robot as an example. In RIKEN-TRI Human-Interactive Robot Research Center, we have developed a nursing-care robot named RIBA (Robot for Interactive Body Assistance) [5-8]. The entire body of RIBA (shown in Figure 2a) is covered with soft protected material. The main task of RIBA is to lift a patient from the bed and transfer her to the chair. By now, it can successfully and comfortable lift and transfer the patient whose maximum weight is $63 \mathrm{~kg}$. The lifting motion (shown in Figure 2b) was designed by the results of [9]. However, like the most robots in the lab environment, the lifting motion is just considered for the special patient and hard to dynamically adjust its motion by different patients. Therefore, RIBA is a good example of adaptive physical human robot interaction based on the physical and social properties of people. In this paper, we try to let the robot detect the physical properties of patient, use the information to modify the interface or motion dynamically and finally improve the PHRI.

This paper introduced the nursing-care robot and the motivation of this research firstly. Then in section 2, a pilot study was designed to explore the relationship between different positions of RIBA arm and patient's comfort and safety. Section 3 is a presentation of equations how to calculate the most suitable positions of RIBA arms. 
Section 4 is a description of the proposed system and methods. Finally we conclude with a discussion of our results, implications for adaptive human robot interaction and directions for future work.
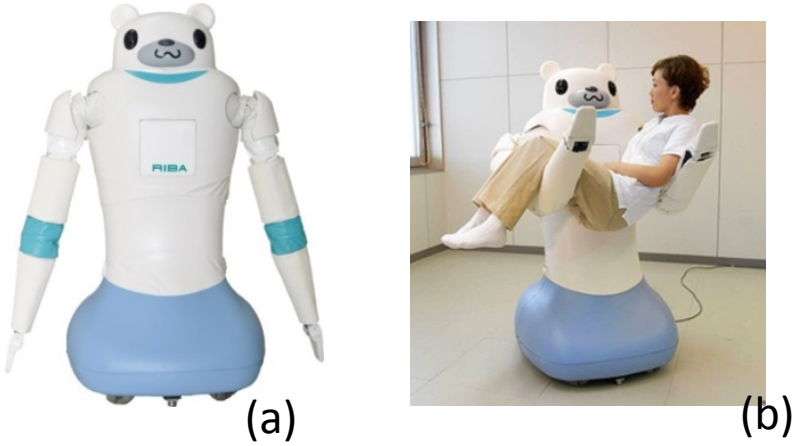

Fig. 2. (a) RIBA; (b) lifting motion

\section{Pilot Study}

Comfort and safety of patient are the most important factors during lifting and transferring. The objective of this experiment is to investigate the relationship between different positions of RIBA arms and patient's comfort and safety. We used two hard covers with four supporting sticks to simulate the RIBA arms (as shown in Figure 3). At the root of supporting stick, force sensor was mounted to measure the supporting force. The independent variable was the height between two arms $(2,8,13,18 \mathrm{~cm})$. The distance between two hard covers was fixed and the number of the distance was $53 \mathrm{~cm}$. Therefore, inclination angle, which is the angle between the link of centers of two arms and horizontal direction, is $2.16,8.58,13.78,18.76$ degrees.

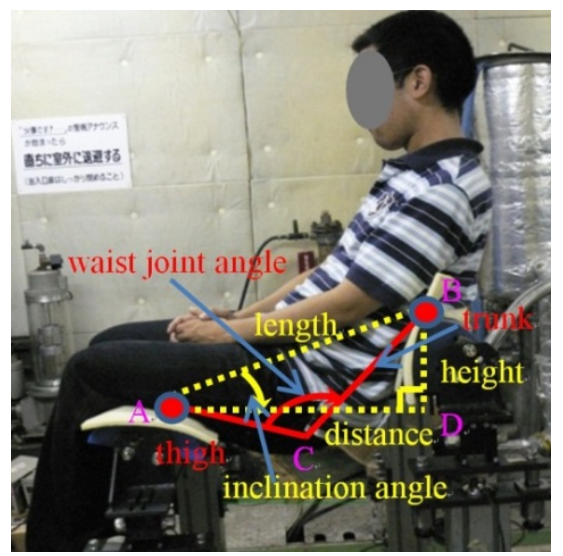

Fig. 3. Experiment setup 
Six male volunteers participated in the experiment. All were not patient and in good health condition. Before the experiment, the task was explained to the participants. They needed to adjust their postures until they felt comfortable and painless and then they were asked to keep that posture for five seconds. Participants were allowed to have a rest between trails. After the experiment, a questionnaire should be completed to rate their subjective preferences by the participants.

To evaluate the workload of trunk and thigh, we measured the supporting forces on two hardcovers. We also recorded the waist joint angle which is the angle between trunk and thigh by different experiment conditions. The overall mean of waist joint angle is 116 degrees (see Figure 4). An ANOVA test showed that there was no significant effect $\left(F_{3,15}=2.679, p>0.05\right)$ from the height between RIBA two arms on the waist joint angle. It suggests that the waist joint angle may be always the same when user feels most comfortable during lifting and transferring. Harrison etc. [10] also explored the relationship between waist angle and user workload in sitting posture. He got the conclusion that user had the lowest dis pressures and lowest electromyography recordings from spinal muscles when the waist joint angle is around 110 to 130 degrees.

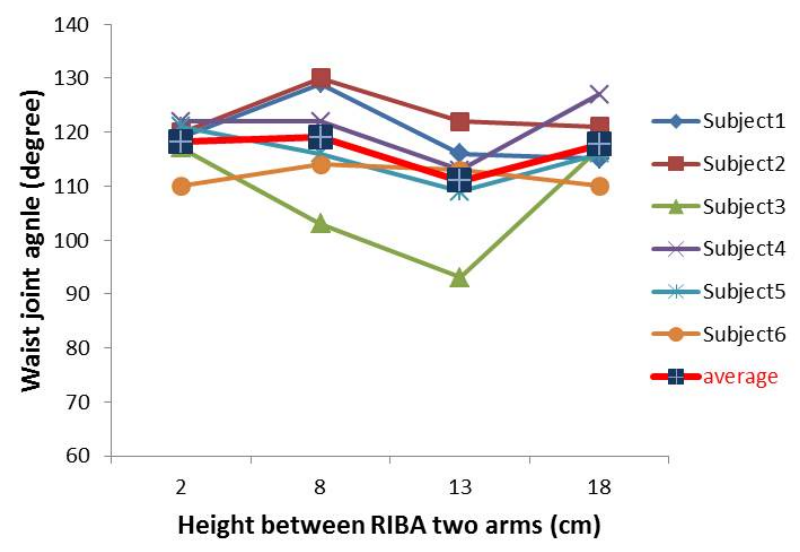

Fig. 4. Waist joint angle by different heights between RIBA arms

According to the results of the questionnaire, the majority of participants (5/6) preferred the setup in which height between RIBA two arms was $13 \mathrm{~cm}$. They reported that "feel comfortable with that posture likes sitting on a chair" and "compared with other conditions, the muscle of necklace is not tight and tired". Results also showed that the main effect of the height between RIBA two arms was statistically significant $(p<0.01)$ on forces of four supporting sticks. With the increase of height between RIBA two arms, the workload of trunk decreased while the one of thigh increased.

In summary, the results of pilot study and reference suggest that when waist joint angle is about 116 degrees, the inclination angle is about 13.78 degrees and lifting posture is similar to the sitting posture, user feels most safe and comfortable. 


\section{Setup of RIBA's Arms}

Generally, the motion and trajectory of robot are set in advance. The final target of lifting robot is that, based on different subjects, custom motion and trajectory of robot can be generated dynamically. In this research, to achieve safe and comfort lifting, it is important to setup the positions of RIBA's arms. Therefore, we analyze the relationship between several parameters of patient and RIBA. As shown in Figure 3, point $\mathrm{A}$ is the center of contact area between thigh and right arm of RIBA, point $\mathrm{B}$ is the center of contact area between trunk and left arm of RIBA, point $\mathrm{C}$ stands for the waist joint position, point $\mathrm{D}$ is the point of a horizontal line passing through $\mathrm{A}$ and a vertical line passing through B. length is the length between two contact points. height is the height between RIBA two arms. distance is the vertical distance between two contact points. thigh is the length between $\mathrm{A}$ and $\mathrm{C}$ and the value of thigh is approximated as half-length of patient's thigh. trunk is the length between B and C and the value of trunk is approximated as half-length of patient's trunk. In the triangle ACB, the angle between thigh and trunk is the waist joint angle. Therefore, we get this equation in the following:

$$
\text { length }=\sqrt[2]{\text { thigh }^{2}+\text { trunk }^{2}-2 \times \text { thigh } \times \text { trunk } \times \cos (\text { waist })}
$$

In the triangle $\mathrm{ABD}$, the angle between length and distance is the inclination angle. And we get these equations in the following:

$$
\begin{aligned}
& \text { height }=\cos (\text { inclination }) \times \text { length } \\
& =\cos (\text { inclination }) \times \sqrt[2]{\text { thigh }^{2}+\text { trunk }^{2}-2 \times \operatorname{thigh} \times \operatorname{trunk} \times \cos (\text { waist })} \\
& \text { distance }=\sin (\text { inclination }) \times \text { length } \\
& =\sin (\text { inclination }) \times \sqrt[2]{\text { thigh }^{2}+\text { trunk }^{2}-2 \times \operatorname{thigh} \times \operatorname{trunk} \times \cos (\text { waist })}
\end{aligned}
$$

In order to life a patient stably and comfortably, height and distance are the most important input parameters of RIBA. As we proved in Section 2, when the inclination angle is 13.78 degrees and waist joint angle is around 116 degrees, patient felt most comfortable. Therefore, in equations (2) and (3), if we know the length of thigh and trunk and then RIBA can adjust the suitable arms' positions by different patients.

\section{System and Methods}

Human behavior analysis [12-14] has been a long focus of interest on computer vision and machine learning areas. Most of researchers used motion capture device and laser scanners to analyze the depth maps from the real world. However, these devices are very expensive and hard to be mounted on the robot. Kinect, which is a low-cost device, uses a light technique [11] to generate depth map which is made of discrete point cloud. It is a motion sensing input device [15] developed by Microsoft that enables users to naturally interact with games and other programs without the need to physically touch a 
game controller or object of any kind. It achieves this through a natural user interface by tracking the user's body movement and by using gestures and spoken commands. Although Microsoft Kinect can estimate different poses and detect 3D positions of body parts, it just can recognize the people skeleton in sitting and standing posture. PCL [16] is an open source and we can use this source to grab the point cloud in the real time. By considering for the task of lifting a patient from bed, our target is to recognize the patient position, the lengths of thigh and trunk. In the following, we propose an approach to achieve this target by PCL and other algorithms.
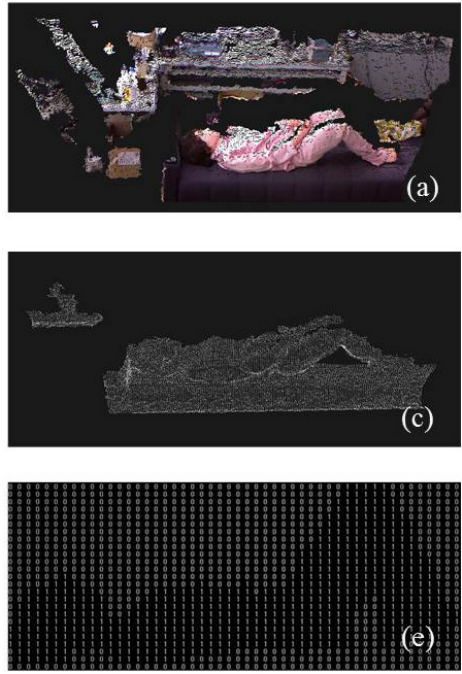
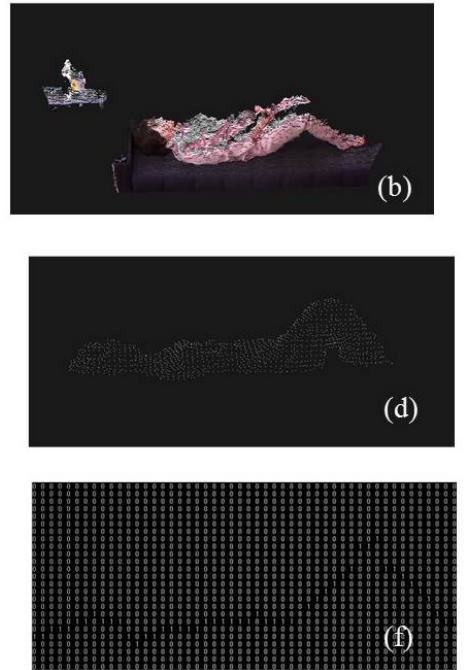

Fig. 5. Graphical illustration of the process. (a) pass filter in $Y$ direction(a) pass filter in $Z$ direction (c) voxel grid down sampling (d) segmented body (e) organized point cloud (f) after thinning method.

Firstly, pass filter was used to reduce the number of points in $\mathrm{Y}$ and $\mathrm{Z}$ directions (see Figure 5a, 5b). In order to do the down sampling, 3D voxel grid filter was applied and the leaf size was $1 \mathrm{~cm}$ (see Figure 5c). Then we tried to detect the plane which was the bed plane from the rest point cloud, the patient must lie on that bed and finally we can find the patient. Similar like the way to detect the objects on the table [17], random sample consensus algorithm was used to detect the plane and the area of bed is recognized. And then, all the points above the bed from $1 \mathrm{~cm}$ to $50 \mathrm{~cm}$ were extracted. Finally, clustering algorithm was used to get the biggest cluster point set which was the segmentation of patient body (see Figure $5 \mathrm{~d}$ ). The patient point cloud was then transferred from $3 \mathrm{D}$ to $2 \mathrm{D}$ and we used the distance information to organize the $2 \mathrm{D}$ point cloud into binary array. " 1 " means the points belonging to the patient are in this area, "0" means the points belonging to the patient are not in this area (see Figure 5e). Simple thinning method in which calculates the center of column array was used to find the skeleton of patient (see Figure 5f). On the next step, linear least squares regression is used to model the three lines which stand for the trunk, thigh and shank. The joints of them are the knee position and waist position. Then, we can get the length of trunk and thigh. 


\section{Conclusion and Future Works}

The concept of adaptive user-center interface for safe and comfort in PHRI was proposed. The robot can dynamically adjust its motion and trajectory based on the social and physical properties of user. In this paper, we took a nursing-care robot RIBA as an example and explored what and how the physical properties of patient affected the setup of RIBA's arms. The results of experiment showed the waist joint angle may be always the same when user feels most comfortable during lifting and transferring. After analyzing the equations between the parameters of RIBA and patient, we found that the setup of RIBA's arms should be changed by the different length of trunk and thigh of patient. Finally a method with Kinect of detecting trunk and thigh was proposed. This paper is an attempt to explore the adaptive human robot interaction and contributes to provide a more complete vocabulary in this direction that adaptive interface researcher of human robot interaction can leverage.

In the future, we plan to further investigate how incorporate the physical and social properties of people in interaction between human and robot. We are also interested in the methods how to detect the properties of people.

\section{References}

1. Aucouturier, J.J., Ikeuchi, K., Hirukawa, H., Nakaoka, S., Shiratori, T., Kudoh, S., Kanehiro, F., Ogata, T., Kozima, H., Okuno, H.G., Michalowski, M.P., Ogai, Y., Ikegami, T., Kosuge, K., Takeda, T., Hirata, Y.: Cheek to chip: dancing robots and AI's future. IEEE Intelligent Systems 23(2) (2008)

2. Minato, T., Yoshikawa, Y., Noda, T., Ikemoto, S., Ishiguro, H., Asada, M.: CB2: a child robot with biomimetic body for cognitive developmental robotics. In: Proceedings of the IEEE/RSJ International Conference on Intelligent Robots and Systems, IROS 2007 (2007)

3. Mitsunaga, N., Miyashita, T., Ishiguro, H., Kogure, K., Hagita, N.: Robovie-IV: a communication robot interacting with people daily in an office. In: Proceedings of the IEEE/RSJ International Conference on Intelligent Robots and Systems, IROS 2006 (2006)

4. Sekmen, A., Challa, P.: Assessment of Adaptive Human-Robot Interactions. KnowledgeBased Systems 42, 49-59 (2013)

5. Mukai, T., Hirano, S., Nakashima, H., Sakaida, Y., Guo, S.: Realization and Safety Measures of Patient Transfer by Nursing-Care Assistant Robot RIBA with Tactile Sensors. Journal of Robotics and Mechatronics 77(782), 116-124 (2011)

6. Mukai, T., Hirano, S., Nakashima, H., Kato, Y., Sakaida, Y., Guo, S., Hosoe, S.: Development of a Nursing-Care Assistant Robot RIBA That Can Lift a Human in Its Arms. In: 2010 IEEE/RSJ International Conference on Intelligent Robots and Systems, IROS 2010, pp. 5996-6001 (2010)

7. Mukai, T., Hirano, S., Yoshida, M., Nakashima, H., Guo, S., Hayakawa, Y.: Tactile-Based Motion Adjustment for the Nursing-Care Assistant Robot RIBA. In: 2011 IEEE International Conference on Robotics and Automation, ICRA 2011, pp. 5435-5441 (2011)

8. Mukai, T., Hirano, S., Yoshida, M., Nakashima, H., Guo, S., Hayakawa, Y.: Whole-Body Contact Manipulation Using Tactile Information for the Nursing-Care Assistant Robot RIBA. In: 2011 IEEE/RSJ International Conference on Intelligent Robots and Systems, IROS 2011, pp. 2445-2451 (2011) 
9. Sakaida, Y., Masuda, H., Chugo, D., Ikeura, R.: Transfer Motion Analysis for Motion Planning of Care Giver Robot. In: ICROS-SICE International Joint Conference 2009, August 18-21, pp. 1650-1653 (2009)

10. Harrison, D., Harrison, S., Croft, A.: Sitting biomechanics, part-1: Review of the literature. J. Manipulative Physiol. Ther. 22(9), 594-609 (1999)

11. Freedman, B., Shpunt, A., Machline, M., Arieli, Y.: Depth mapping using projected patterns. Patent Application 10 (2008); WO 2008/120217 A2

12. Rodgers, J., Anguelov, D., Pang, H.-C., Koller, D.: Object pose detection in range scan data. In: Proc. of IEEE Conf. on Computer Vision and Pattern Recognition (CVPR) (2006)

13. Yang, H., Lee, S.W.: Reconstruction of 3D human body pose from stereo image sequences based on top-down learning. Pattern Recognition 40(11), 3120-3131 (2007)

14. Schwartz, W., Kembhavi, A., Harwood, D., Davis, L.: Human detection using partial least squares analysis. In: ICCV (2009)

15. Shotton, J., Fitzgibbon, A., Cook, M., Sharp, T., Finocchio, M., Moore, R., Kipman, A., Blake, A.: Real-Time Human Pose Recognition in Parts from Single Depth Images. In: CVPR (2011)

16. Point cloud library (pcl), http: / / pointclouds . org/

17. Rusu, R.B.: Semantic 3D Object Maps for Everyday Manipulation in Human Living Environments, PhD Thesis (2009) 